\title{
Change of Number and Size of Blood Platelets in Breast=fed Infants and Intensity of Arakawa's Reaction of Their Mothers on Vitamin B* Administration.
}

r3oth Report of the Peroxidase Reaction.

By

Masayuki Shindo.

(淮 藏 正 之)

(From the Department of Pediatrics, Faculty of Medicine, Tohoku Imperial University, Sendai.

Director: Prof. A. Sato.)

\section{Introduction.}

In the first report on the relation between blood platelets in infants and Arakaw a's reaction of their mothers $\mathrm{I}^{1)}$ reported that, in the cases of infants fed on milk negative to A rakawa's reaction, anemia and thrombocytosis and especially an increase of pathological large platelets were seen, and that blood platelet count was larger generally according as Arakawa's reaction became weaker. In my second report $I^{2)}$ concluded that an increase of blood platelet count was seen in infants fed on milk negative to A rakawa's reaction and that when infants and/or their mothers had been taking a vitamin $B$ preparation for a longer time, blood platelets were within normal limits even in the group of completely or almost completely negative to Arakawa's reaction. And in my third report on the same article, $I^{3}$ ) confirmed, in the 123 cases with no special complications which might influence blood platelets, that the majority of infants fed on human milk completely or almost completely negative to Arakawa's reaction and a part of infants fed on milk of A rakawa's reaction of inter-

* $B_{1}$ is meant throughout the article.

1) M. Shind o, Tohoku J. Exp. Med., 1939, 36, 258.

2) M. Shind o, Tohoku J. Exp. Med., 1939, 37, 45.

3) M. Shind o, Tohoku J. Exp. Med., 1939, 36, 283. 
mediate strength were either in a state of B-avitaminosis or on the brink of B-avitaminosis from the view point of blood platelet count.

According to Ohta and $\mathrm{Suzuki} \mathrm{i}^{4}$ of our Laboratory, a prolongation of the shortest peroxidase stain-time of blood leucocytes, a sign of avitaminosis $B$, will occur in infants with a high blood platelet count which is another sign of avitaminosis $B$.

Later Shiraish $\mathrm{i}$ and $\mathrm{I}^{5)}$ reported that thrombocytosis was seen in B-avitaminotic infants on the study of blood picture of infantile beriberi, infantile preberiberi and B-avitaminotic dyspepsia without any complications.

So, if an increase of blood platelet count is due to B-avitaminosis in the cases of infants fed on milk negative to Arakawa's reaction, it will be expected that platelet count will decrease to the normal on an administration of vitamin $B$.

In the present paper, therefore, I set out to study the qualitative and quantitative change of blood platelets in infants on vitamin $\mathrm{B}$ administration.

\section{Method of Experiment.}

1. Materials. Breast-fed infants who visited our Pediatric Clinic from summer 1934 to autumn 1937.

2. Method used. Thrombocytes were counted according to the method of $\mathrm{Fonio}^{6}$ with a slight modification. After cleaning with alcohol and then ether, a cut was made on the ear-lobe and the first drop of blood was mixed with a drop of $14 \% \mathrm{MgSO}_{4}$ by use of a glass-rod. And a couple of films were made on cover glass at one time. After these were air-dried, they were fixed with methyl alcohol for 3 minutes, and then stained with Giemsa ( 2 drops of the stain in 1 c.c. of distilled water) as long as 10 hours. Horwitz's ${ }^{7}$ classification of platelet was adopted.

a. Small platelets up to $1 / 4$ diameter of red cell.

b. Middle-sized platelets from $1 / 4$ to $2 / 3$ of the diameter of red cell.

c. Large platelets from $2 / 3$ to $1 / 1$ of the diameter of red cell.

3. Administration of vitamin $B$. All babies were treated with vitamin B perorally, though some of them were also injected* with it subcutaneously at the same time. As to their mothers, in all cases except for 6 cases vitamin $B$ was administered per os.

* Immediate influence of the injection on blood platelets was out of the question, for the interval of blood examination was 7-10 days.

4) F. Ohta and T. Suzuki, Tohoku J. Exp. Med., 1937, 31, 247.

5) Sh. Shiraishi and M. Shind o, Tohoku J. Exp. Med., 1938, 32, 470.

6) A. Fon io, Dtsch. Z. Chir., 1912, 102, 178.

7) S. Horwits, Kl. Wsehr., 1931, 10, 1613. 
International units of vitamin B preparations used are as follows :- " Injectio Oryzanin Fortior" of Sankyo \& Co. is the vitamin $B_{1}$ preparations contained in 1 c.c. $0.5 \mathrm{mg}$. of $B_{1}$ crystals $=333$ international units. Powder of Oryzanin and Beriberol (Beriberol purum is ten times as potent as Beriberol) 100 grms. $=4,000$ and 608 international units respectively. Liquor Oryzanin and Liq. Beriberol 100 c.c. $=600$ and 102 international units.

4. Detoxicating hormone of the liver, yakriton.

Over half the cases of babies and in some cases of mothers yakriton, which will mobilize vitamin $B$ according to Chiba and $A b^{8)}$ and Takamatsu, ${ }^{9}$ was injected subcutaneously.

\section{Results.}

I. Infuence of Vitamin $B$ with and without Use of Yakriton on Number and Size of Blood Platelets in the Cases of Infantile Beriberi, Infantile Preberiberi, B-avitaminotic Dyspepsia and so-called Human milk Intoxication.

Results of 6 cases of infantile beriberi, 6 cases of infantile preberiberi, 8 cases of B-avitaminotic dyspepsia and one case of so-called human milk intoxication were tabulated in Table 1 . To babies vitamin $B$ preparation was administered perorally in all cases and subcutaneously in only 4 cases, while to mothers in 3 cases (Nos. 9, 12 and 19) no vitamin $B$ preparation was administered.

Yakriton, the detoxicating hormone of the liver, was injected in 11 cases of babies and in 3 cases of mothers.

In 6 cases (Nos. 2, 5, 7, 12, 15 and 18) A rakawa's reaction of mother's milk and blood of infants were examined 3 times and in the others twice. The interval between the two examinations was about $7-10$ days.

At the beginning of experiment, A rakaw a's reaction of mothers milk was completely or almost completely negative in 12 cases and the reaction was clinically negative in the other cases too. On an administration of vitamin $B, A$ ra ka wa's reaction became clinically positive $^{*}$ in Nos. $1,3,7,15,17$ and 21 , or at least their milk increased in

* "Arakawa-positive" may be used in two different senses. One of these is: Arakawa-positive in a biochemical sense. A sample of human milk is said to have become Araka wa-positive, when it became blue on the addition of A rakawa's reagent. The other of these two senses is: Arakawa-positive in clinical sense. A sample of human milk is clinically Arakawa-positive only when it shows sueh a reaction as + or $H$ in one minute of the addition of A rakawa's reagent.

8) M. Chiba and J. Abe, Zika Zasshi, 1931, 1431.

9) A. Takamatsu, Tohokn J. Exp. Med., 1934, 23, 372. 
intensity of the reaction. And it is to be noticed that Arakawa's reaction did not change in all the 3 cases (Nos. 9, 12 and 19 in Table 1) in which vitamin $B$ was not administered to their mothers.

In 13 cases red cell count was reduced, the minimal value of 4,000 thousands was seen in No. 17 and red cell count showed an increase in 12 cases on vitamin $B$ administration.

In all the 21 cases of B-avitaminosis in Table 1 , a decrease of thrombocytes was seen in parallelism with an increase in intensity of Arakawa's reaction on vitamin $B$ administration. As to blood platelet count, in 2 cases (Nos. 2 and 5) platelets began to decrease after showing a temporary increase of them, in 1 case (No. 12 in Table 2) thrombocytes became normal in number after a temporary decrease to a subnormal count, and in the remaining 3 cases (Nos. 7, 15 and 18) platelets decreased gradually. In most cases platelet count returned to normal within from 7 to 10 days and in all the 6 cases in which Arakawa's reaction became clinically positive, platelets reached a value within normal limits.

It is to be noticed that platelet count returned to normal in Nos. $11,12,13$ and 18 in spite of the fact that it remained negative to Arakawa's reaction on an administration of vitamin $B$. This reminds one of Takamatsu ${ }^{10}$ who said that when mothers with Arakawanegative milk were taking vitamin $B$, the milk was losing in its toxicity in spite of the even remaining negative reaction.

The classification of platelets was made in 16 cases out of all 21 cases. Large platelets were over $5 \%$ in 7 cases and in these cases small platelets were within normal limits (19-24\%) in 5 cases. At the beginning of vitamin $B$ administration, large platelets showed a gradual decrease and small platelets began to increase. In 7 cases (Nos. $3,5,6,9,11,12$ and 14 in Table 1 ) the ratio-small : middle: large-returned to normal, but in the remaining 9 cases small platelets were yet larger than $24 \%$ while large platelets were within normal limits. The decrease of platelets was not remarkable in 2 cases (Nos. 9 and 19 in Table 1 ) out of all 3 cases in which vitamin $B$ had not been administered to mothers.

In 8 cases ( $73 \%$ ) out of 11 cases in which yakriton (1/2 R.A.U., every other day) was administered to infants subcutaneously, platelet count returned to normal, while in 7 cases (70\%) out of 10 cases in which the hormone was not given, platelet count decreased indeed, but

10) A. Takamats u, Tohoku J. Exp. Med., 1934, 23, 46. 
TABLE 1. Influenoe of vitamin $B$ on number and size of blood platelet, infantile preberiberi, B-avitaminotic dyspepsia

\begin{tabular}{|c|c|c|c|c|c|c|}
\hline \multirow{3}{*}{$\begin{array}{l}\text { Case } \\
\text { No. }\end{array}$} & \multirow{3}{*}{ Date } & \multirow{3}{*}{ Name } & \multirow{3}{*}{ Sex } & \multirow{3}{*}{$\begin{array}{c}\text { Age } \\
(\mathrm{m}= \\
\text { months, } \\
\mathrm{d}=\text { days })\end{array}$} & \multirow{3}{*}{ Diagnosis } & Arakawa's \\
\hline & & & & & & Right \\
\hline & & & & & & $0^{\prime} 1^{\prime} 2^{\prime} 3^{\prime} 4^{\prime} 5^{\prime}$ \\
\hline 1 & $\begin{array}{r}\text { Ang. } 13 \\
22 \\
\end{array}$ & I. $\mathbf{H}$. & 古 & $\begin{array}{ll}49 & d \\
58 & d\end{array}$ & Infantile beriberi & $\bar{H}-\bar{H}-\bar{H}-\bar{H}$ \\
\hline 2 & \begin{tabular}{|r|} 
Jun. 16 \\
\\
23 \\
Jul. $\quad 3$ \\
\end{tabular} & H.H. & 우 & $5 \mathrm{~m}$ & $\begin{array}{l}\text { Infantile beriberi with } \\
\text { hypotrophy }\end{array}$ & $\begin{array}{l}- \pm++++ \\
-++H+H \\
+H+H+H\end{array}$ \\
\hline 3 & $\begin{array}{r}\text { Apr. } 19 \\
28 \\
\end{array}$ & Y.O. & 우 & $7 \mathrm{~m}$ & $\begin{array}{l}\text { Infantile beriberi with } \\
\text { hypotrophy }\end{array}$ & $\begin{array}{l} \pm \pm \pm \pm \pm \\
\pm \#+\# \#+H\end{array}$ \\
\hline 4 & $\begin{aligned} \text { Feb. } 15 \\
26\end{aligned}$ & Y.S. & 우 & $\begin{array}{ll}50 & d \\
61 & d\end{array}$ & Infantile beriberi & $=-\ldots-1$ \\
\hline 5 & $\begin{array}{ll}\text { Jun. } & 11 \\
21 \\
\text { Jul. } & 16 \\
\end{array}$ & T. 0 & 今 & $\begin{array}{r}40 \mathrm{~d} \\
50 \mathrm{~d} \\
2 \mathrm{~m}\end{array}$ & Infantile beriberi & $\begin{array}{l}- \pm \pm \pm \pm \pm \\
- \pm \pm++t \\
-- \pm- \pm \pm\end{array}$ \\
\hline 6 & $\begin{array}{ll}\text { May } 13 \\
\\
21\end{array}$ & T.A. & 우 & $4 \mathrm{~m}$ & Infantile beriberi & $\begin{array}{l}- \pm+++ \\
- \pm+++4\end{array}$ \\
\hline 7 & $\begin{array}{r}\text { Nov. } 15 \\
26 \\
\text { Dee. } 7 \\
\end{array}$ & Y.K. & ㅇ. & $\begin{array}{ll}62 & \mathrm{~d} \\
73 & \mathrm{~d} \\
84 & \mathrm{~d} \\
\end{array}$ & Infantile preberiberi & $\begin{array}{l}- \pm \pm \pm \pm \pm \\
\pm \pm+ \pm H \\
\pm+H \#+H\end{array}$ \\
\hline 8 & $\begin{array}{r}\text { Sep. } \\
23 \\
28 \\
\end{array}$ & M.E. & 우 & $9 \mathrm{~m}$ & Infantile preberiberi & $\begin{array}{l}- \pm \pm \pm+1 \\
- \pm+4+H\end{array}$ \\
\hline 9 & $\begin{array}{rr}\text { Sep. } 25 \\
\text { Oct. } \quad 2 \\
\end{array}$ & S. $\dot{K}$. & 今 & $\begin{array}{ll}35 & d \\
42 d & \end{array}$ & Infantile preberiberi & $\begin{array}{l}-- \pm \pm+ \\
--- \pm \pm+\end{array}$ \\
\hline 10 & $\begin{aligned} \text { Feb. } 19 \\
28\end{aligned}$ & S.M. & $\delta$ & $\begin{array}{l}81 \mathrm{~d} \\
90 \mathrm{~d} \\
\end{array}$ & $\begin{array}{l}\text { Infantile preberiberi } \\
\text { + hernia umbilicalis }\end{array}$ & $\begin{array}{l}-- \pm \pm \pm \\
- \pm+ \pm+H\end{array}$ \\
\hline 11 & $\begin{array}{r}\text { Apr. } 19 \\
28 \\
\end{array}$ & I. M. & $\delta$ & $2 \mathrm{~m}$ & Infantile preberiberi & $\begin{array}{l}-+++1+ \\
-- \pm \pm \pm\end{array}$ \\
\hline 12 & \begin{tabular}{|r|} 
Oct. \\
16 \\
22 \\
\end{tabular} & J.I. & 古 & $\begin{array}{l}60 \mathrm{~d} \\
69 \mathrm{~d} \\
75 \mathrm{~d}\end{array}$ & $\begin{array}{l}\text { Infantile preberiberi } \\
\text { with hypotrophy }\end{array}$ & $\begin{array}{l}--- \pm \pm \pm \\
-----1 \\
------ \\
\end{array}$ \\
\hline 13 & $\begin{array}{l}\text { Sep. } 20 \\
\text { Oet. } \quad 2 \\
\end{array}$ & S. S. & $\delta$ & $\begin{array}{l}54 \mathrm{~d} \\
66 \mathrm{~d} \\
\end{array}$ & $\begin{array}{c}\text { B-avitaminotic } \\
\text { dyspepsia }\end{array}$ & $\begin{array}{l}- \pm \pm \pm+ \\
-- \pm \pm+\end{array}$ \\
\hline 14 & \begin{tabular}{|l|r|} 
Nov. 22 \\
Dec. $\quad 2$ \\
\end{tabular} & R.S. & 今 & $12 \mathrm{~m}$ & $\begin{array}{c}\text { B-avitaminotic } \\
\text { dyspepsia }\end{array}$ & $\begin{array}{l}-- \pm-- \\
- \pm \pm \pm \pm \pm\end{array}$ \\
\hline 15 & 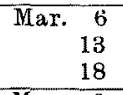 & T.K. & 今 & $9 \mathrm{~m}$ & $\begin{array}{c}\text { B-avitaminotie } \\
\text { dyspepsia }\end{array}$ & $\begin{array}{l} \pm++H H H \\
\pm H+H H H \\
\pm H+H \#+4\end{array}$ \\
\hline 16 & $\begin{array}{r}\text { May } 8 \\
22 \\
\end{array}$ & $\mathrm{~K}, \mathrm{~T}$. & $\widehat{\delta}$ & $5 \mathrm{~m}$ & $\begin{array}{l}\text { B-avitaminotic } \\
\text { dyspepsia }\end{array}$ & $\begin{array}{l}----- \\
-----\end{array}$ \\
\hline 17 & $\begin{array}{rr}\text { Jul. } & 13 \\
\text { Aug. } & 8 \\
\end{array}$ & E.M. & s & $\begin{array}{l}4 \mathrm{~m} \\
5 \mathrm{~m}\end{array}$ & $\begin{array}{c}\text { B-avitaminotic } \\
\text { dyspepsia }\end{array}$ & $\begin{array}{l}-\overline{-}-\overline{-}-\overline{-} \\
-H+H+H\end{array}$ \\
\hline 18 & $\begin{array}{r}18 \\
25 \\
30 \\
\end{array}$ & J.T. & $\hat{\delta}$ & $4 \mathrm{~m}$ & $\begin{array}{c}\text { B-avitaminotic } \\
\text { dyspepsia }\end{array}$ & $\begin{array}{l}- \pm++t \\
-- \pm-1+1 \\
- \pm \pm++t\end{array}$ \\
\hline 19 & $\begin{array}{r}\text { Sep. } 18 \\
25 \\
\end{array}$ & K.G. & 古 & $12 \mathrm{~m}$ & $\begin{array}{l}\text { B-avitaminotic } \\
\text { dyspepsia }\end{array}$ & $\begin{array}{l}- \pm++4 \\
- \pm--1\end{array}$ \\
\hline 20 & $\begin{array}{lr}\text { Sep. } & 23 \\
\text { Oct. } & 2 \\
\end{array}$ & T.S. & 우 & $4 \mathrm{~m}$ & $\begin{array}{c}\text { B-avitaminotic } \\
\text { dyspepsia }\end{array}$ & $\begin{array}{l}----- \pm \\
-----1\end{array}$ \\
\hline 21 & $\begin{array}{l}\text { Jul. } 31 \\
\text { Aug. } \quad 5\end{array}$ & A.T. & s & $9 \mathrm{~m}$ & $\begin{array}{l}\text { "Intoxication of human } \\
\text { milk" }\end{array}$ & $\pm \bar{H}-\bar{H}-\bar{H}$ \\
\hline
\end{tabular}


red cell count and Arakawa's reaction in the cases of infantile beriberi, and so-ealled human milh intoxication.

\begin{tabular}{|c|c|c|c|c|c|c|}
\hline \multirow{3}{*}{$\frac{\frac{\text { reaction }}{\text { Left }}}{0^{\prime} 1^{\prime} 2^{\prime} 3^{\prime} 4^{\prime} 5^{\prime}}$} & \multirow{3}{*}{$\begin{array}{c}\text { Red cell } \\
\text { count per } \\
\text { cmm. } \\
\text { (thou- } \\
\text { sands) }\end{array}$} & \multirow{3}{*}{$\begin{array}{l}\text { Total } \\
\text { count per } \\
\text { cmm. } \\
\text { (thou- } \\
\text { sands) }\end{array}$} & \multicolumn{3}{|c|}{ Blood platelets } & \multirow{2}{*}{$\frac{\text { Administration }}{\text { To }}$} \\
\hline & & & \multicolumn{3}{|c|}{ Classification } & \\
\hline & & & Small & Middle & Large & Per os daily (grs.) \\
\hline$\overline{-}-\bar{H}-\bar{H}-\bar{H}$ & $\begin{array}{l}4,600 \\
4,700\end{array}$ & $\begin{array}{l}510 \\
270 \\
\end{array}$ & $\begin{array}{l}20 \\
27\end{array}$ & $\begin{array}{l}71 \\
70\end{array}$ & $\begin{array}{l}9 \\
3\end{array}$ & B. p. \\
\hline $\begin{array}{l}- \pm+ \pm+ \pm \\
\pm \pm \pm \pm+ \pm\end{array}$ & $\begin{array}{l}4,700 \\
4,550 \\
4,980\end{array}$ & $\begin{array}{l}423 \\
441 \\
353\end{array}$ & $\begin{array}{l}30 \\
34 \\
25\end{array}$ & $\begin{array}{l}67 \\
66 \\
74\end{array}$ & $\begin{array}{l}3 \\
0 \\
1\end{array}$ & $\begin{array}{ll}\text { Liq. ory. } & 5.0 \\
\text { Ebios } & 0.3\end{array}$ \\
\hline 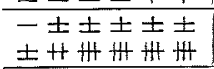 & $\begin{array}{l}5,360 \\
6,130 \\
\end{array}$ & $\begin{array}{l}713 \\
356 \\
\end{array}$ & $\begin{array}{l}- \\
23 \\
22 \\
\end{array}$ & $\begin{array}{l}74 \\
78\end{array}$ & $\begin{array}{l}3 \\
0 \\
0\end{array}$ & Liq. ory. 5.0 \\
\hline $\begin{array}{l}-- \pm \pm \pm+ \\
-- \pm \pm \pm+\end{array}$ & $\begin{array}{l}4,150 \\
5,180\end{array}$ & $\begin{array}{l}575 \\
394 \\
\end{array}$ & $\begin{array}{l}19 \\
27 \\
\end{array}$ & $\begin{array}{l}77 \\
72 \\
\end{array}$ & $\begin{array}{l}4 \\
1 \\
\end{array}$ & $\begin{array}{ll}\text { Liq. ory. } & 5.0 \\
\text { B. p. } & 0.03 \\
\end{array}$ \\
\hline $\begin{array}{l}\overline{-}-\overline{-}-\bar{H} \\
\pm+H+H \\
-+H+H\end{array}$ & $\begin{array}{l}4,160 \\
3,610 \\
3,330 \\
\end{array}$ & $\begin{array}{l}359 \\
492 \\
253 \\
\end{array}$ & $\begin{array}{l}28 \\
35 \\
22 \\
\end{array}$ & $\begin{array}{l}72 \\
64 \\
78\end{array}$ & $\begin{array}{l}0 \\
1 \\
0\end{array}$ & $\begin{array}{ll}\text { Liq. ory. } & 5.0 \\
\text { Ebios } & 0.5\end{array}$ \\
\hline $\begin{array}{l}- \pm \pm \pm \pm \pm \\
- \pm+++4\end{array}$ & $\begin{array}{l}4,860 \\
4,760 \\
\end{array}$ & $\begin{array}{l}435 \\
433 \\
\end{array}$ & $\begin{array}{l}21 \\
24 \\
\end{array}$ & $\begin{array}{l}70 \\
72 \\
\end{array}$ & $\begin{array}{l}9 \\
4 \\
\end{array}$ & Liq. ory. 3.0 \\
\hline $\begin{array}{l}------ \\
-- \pm \pm \pm \pm \\
-+H+H+H\end{array}$ & $\begin{array}{l}4,030 \\
4,260 \\
4,440 \\
\end{array}$ & $\begin{array}{l}323 \\
290 \\
242 \\
\end{array}$ & $\begin{array}{l}25 \\
35 \\
31 \\
\end{array}$ & $\begin{array}{l}72 \\
63 \\
69 \\
\end{array}$ & $\begin{array}{l}3 \\
2 \\
0\end{array}$ & $\begin{array}{ll}\text { Liq. ory. } & 3.0 \\
\text { B. p. } & 0.03 \\
\end{array}$ \\
\hline$-\overline{-}-\bar{H}-\bar{H}$ & $\begin{array}{l}4,530 \\
4,910\end{array}$ & $\begin{array}{l}472 \\
411\end{array}$ & $\begin{array}{l}23 \\
28\end{array}$ & $\begin{array}{l}70 \\
68\end{array}$ & $\begin{array}{l}7 \\
3\end{array}$ & $\begin{array}{ll}\text { Liq. spel. } & 5.0 \\
\text { B. p. } & 0.03\end{array}$ \\
\hline $\begin{array}{c}-- \pm \pm+ \\
- \pm++t+4\end{array}$ & $\begin{array}{l}4,100 \\
3,980\end{array}$ & $\begin{array}{l}553 \\
464 \\
\end{array}$ & $\begin{array}{l}22 \\
20 \\
\end{array}$ & $\begin{array}{l}73 \\
77\end{array}$ & $\begin{array}{l}5 \\
\mathbf{5}\end{array}$ & $\begin{array}{ll}\text { Liq. ory. } & 3.0 \\
\text { B. p. } & 0.03\end{array}$ \\
\hline $\begin{array}{l}-1 \pm \pm \pm \pm \\
- \pm \pm \pm \pm\end{array}$ & $\begin{array}{l}5,300 \\
5,800\end{array}$ & $\begin{array}{l}830 \\
560\end{array}$ & - & - & - & $\begin{array}{lc}\text { Liq. Berib. } 5.0 \\
\text { B. p. } & 0.03\end{array}$ \\
\hline-++++1 & $\begin{array}{l}5,190 \\
4,030\end{array}$ & $\begin{array}{l}368 \\
258 \\
\end{array}$ & $\begin{array}{l}20 \\
24\end{array}$ & $\begin{array}{l}76 \\
76 \\
\end{array}$ & $\begin{array}{l}4 \\
0\end{array}$ & $\begin{array}{ll}\text { Liq. ory. } & 3.0 \\
\text { B. p. } & 0.03\end{array}$ \\
\hline $\begin{array}{l}-- \pm \pm \pm \pm \\
- \pm \pm+ \pm+ \\
----1\end{array}$ & $\begin{array}{l}4,100 \\
4,530 \\
4,750\end{array}$ & $\begin{array}{l}260 \\
193 \\
230\end{array}$ & $\begin{array}{l}19 \\
23 \\
23\end{array}$ & $\begin{array}{l}78 \\
75 \\
77 \\
\end{array}$ & $\begin{array}{l}3 \\
2 \\
0\end{array}$ & $\begin{array}{l}\text { Liq. Berib. } 5.0 \\
\text { B. p. } \quad 0.05\end{array}$ \\
\hline $\begin{array}{l}-\overline{-}-\overline{+} \\
- \pm \pm++\end{array}$ & $\begin{array}{l}4,610 \\
4,620\end{array}$ & $\begin{array}{l}511 \\
280 \\
\end{array}$ & $\begin{array}{l}24 \\
34 \\
\end{array}$ & $\begin{array}{l}73 \\
67 \\
\end{array}$ & $\begin{array}{l}3 \\
2 \\
\end{array}$ & Vitamin $B(+)$ \\
\hline $\begin{array}{l}-----\overline{-} \\
- \pm \pm \pm++\end{array}$ & $\begin{array}{l}4,050 \\
5,800\end{array}$ & $\begin{array}{l}606 \\
480\end{array}$ & $\begin{array}{l}23 \\
24 \\
\end{array}$ & $\begin{array}{l}70 \\
73 \\
\end{array}$ & $\begin{array}{l}7 \\
3 \\
\end{array}$ & Liq. ory. 3.0 \\
\hline $\begin{array}{l} \pm++H+H \\
\pm H+H H+H \\
\pm H+H H H H\end{array}$ & $\begin{array}{l}6,850 \\
6,950 \\
5,550\end{array}$ & $\begin{array}{l}780 \\
750 \\
270 \\
\end{array}$ & $\begin{array}{l}- \\
- \\
\end{array}$ & $\begin{array}{l}- \\
- \\
\end{array}$ & $\begin{array}{l}- \\
- \\
-\end{array}$ & $\begin{array}{lc}\text { Liq. Berib. } 5.0 \\
\text { B. p. } & 0.03 \\
\end{array}$ \\
\hline-- \pm- \pm+ \pm & $\begin{array}{l}5,000 \\
4,880\end{array}$ & $\begin{array}{l}520 \\
421\end{array}$ & - & - & - & $\begin{array}{ll}\text { Liq. Berib. } 5.0 \\
\text { B. p. } \quad 0.03\end{array}$ \\
\hline$-\overline{-}-\bar{H}-\bar{H}$ & $\begin{array}{l}4,000 \\
4,570\end{array}$ & $\begin{array}{l}380 \\
230\end{array}$ & - & - & - & $\begin{array}{ll}\text { Liq. Berib. } 5.0 \\
\text { B. p. } & 0.03\end{array}$ \\
\hline $\begin{array}{l}- \pm \pm++t \\
- \pm \pm \pm++ \\
- \pm \pm+++\end{array}$ & $\begin{array}{l}4,300 \\
4,450 \\
4,510\end{array}$ & $\begin{array}{l}637 \\
451 \\
313\end{array}$ & $\begin{array}{l}34 \\
31 \\
25 \\
\end{array}$ & $\begin{array}{l}59 \\
68 \\
74\end{array}$ & $\begin{array}{l}7 \\
1 \\
1\end{array}$ & Liq. Berib. 5.0 \\
\hline $\begin{array}{l}- \pm+++H \\
- \pm++++\end{array}$ & $\begin{array}{l}5,240 \\
5,140\end{array}$ & $\begin{array}{l}688 \\
502\end{array}$ & $\begin{array}{l}19 \\
38\end{array}$ & $\begin{array}{l}76 \\
64\end{array}$ & $\begin{array}{l}5 \\
3\end{array}$ & Liq. ory. 3.0 \\
\hline------ & $\begin{array}{l}3,820 \\
4,260\end{array}$ & $\begin{array}{l}502 \\
423\end{array}$ & $\begin{array}{l}26 \\
30\end{array}$ & $\begin{array}{l}70 \\
66\end{array}$ & $\begin{array}{l}4 \\
4 \\
\end{array}$ & B. p. \\
\hline$-\overline{-}-\overline{+}-\bar{H}+4$ & $\begin{array}{l}6,350 \\
5,190\end{array}$ & $\begin{array}{l}533 \\
331\end{array}$ & - & - & - & $\begin{array}{ll}\text { Liq. Berib. } 5.0 \\
\text { B. p. } \quad 0.05\end{array}$ \\
\hline
\end{tabular}

Ory. = Oryzanin (powder), Liq. Berib. $=$ Liquor Beriberol, B. p. = Beriberolum purum

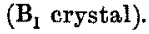


Table 1. Continued.

\begin{tabular}{|c|c|c|c|c|c|}
\hline \multicolumn{3}{|l|}{ of vitamin $B *$} & \multirow{2}{*}{\multicolumn{2}{|c|}{$\begin{array}{l}\text { Subeutaneous injec- } \\
\text { tion of yakriton } \\
\text { (R.A.U.) } \\
\text { every other day }\end{array}$}} & \multirow{3}{*}{ Remarks } \\
\hline sucklings & \multicolumn{2}{|c|}{ To mothers } & & & \\
\hline $\begin{array}{c}\text { By injection } \\
\text { every other day }\end{array}$ & $\begin{array}{c}\text { Per os daily } \\
\text { (grs.) }\end{array}$ & $\begin{array}{c}\text { By injection } \\
\text { every other day }\end{array}$ & $\begin{array}{c}\text { To } \\
\text { sucklings }\end{array}$ & $\begin{array}{c}\text { To } \\
\text { mothers }\end{array}$ & \\
\hline $0.5 \mathrm{mg}$. & Liq. oryz. 30.0 & $\overline{-}$ & $1 / 2$ & - & \\
\hline $\begin{array}{l}- \\
- \\
-\end{array}$ & Vitamin B $(+)$ & $\overline{-}$ & $\begin{array}{l}- \\
-\end{array}$ & $\overline{-}$ & \\
\hline- & $\begin{array}{ll}\text { Ory. } & 5.0 \\
\text { Ebios } & 3.0\end{array}$ & - & $1 / 2$ & - & 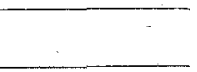 \\
\hline- & $\begin{array}{ll}\text { Ory. } & 5.0 \\
\text { Ebios } & 3.0 \\
\end{array}$ & - & - & $1 / 2$ & . \\
\hline $0.5 \mathrm{mg}$. & Vitamin $B(+)$ & - & $\begin{array}{l}- \\
- \\
-\end{array}$ & 1 & 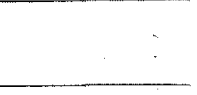 \\
\hline Spelzon 5.0 & Liq. ory. 40.0 & Spelzon $\quad 5.0$ & $1 / 2$ & & \\
\hline $\begin{array}{l}- \\
-\end{array}$ & Liq. ory. 20.0 & $\overline{-}$ & $1 / 2$ & $\begin{array}{l}- \\
- \\
\end{array}$ & \\
\hline- & Vitamin B $(+)$ & - & - & - & \\
\hline- & - & - & - & - & \\
\hline - & Liq. ory. 20.0 & - & $1 / 2$ & - & \\
\hline- & Ebios $\quad 6.0$ & - & + & - & \\
\hline $\begin{array}{l}- \\
- \\
-\end{array}$ & $\begin{array}{l}- \\
- \\
-\end{array}$ & $\begin{array}{l}- \\
- \\
\end{array}$ & $1 / 2$ & $\begin{array}{l}- \\
-\end{array}$ & $\begin{array}{l}\text { Mother's milk } \\
\text { and cow's milk. }\end{array}$ \\
\hline - & Vitamin $\mathrm{B}(+)$ & - & $1 / 2$ & - & \\
\hline- & Liq. ory. 20.0 & - & - & - & \\
\hline $\begin{array}{l}- \\
-\end{array}$ & Liq. ory. 20.0 & - & $1 / 2$ & $\begin{array}{l}- \\
- \\
\end{array}$ & \\
\hline- & Liq. ory, 20.0 & $\underline{-}$ & - & - & \\
\hline$\overline{-}$ & Liq. ory. 20.0 & - & $1 / 2$ & - & 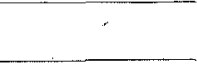 \\
\hline $\begin{array}{l}- \\
-\end{array}$ & Liq. ory. 30.0 & $\begin{array}{l}- \\
- \\
-\end{array}$ & $1 / 2$ & $1 / 2$ & . \\
\hline- & - & - & - & - & \\
\hline- & Liq. ory. 20.0 & - & - & $\overline{-}$ & \\
\hline $0.5 \mathrm{mg}$. & $\begin{array}{c}\text { Liq. ory. } 20.0 \\
\text { Liq. Berib. } 20.0\end{array}$ & $0.5 \mathrm{mg}$. & $1 / 2$ & $\overline{-}$ & \\
\hline
\end{tabular}


did not attain the normal count. In all these cases vitamin $B$ was given, so it is very probable that yakriton will act to mobilize vitamin $B$ in its effect.

II. Influence of Vitamin $B$ with or without Use of Yakriton on Number and Size of Blood Platelets in Such Diseases as with no* Influence on the Platelet Count or in Such Diseases as accompanied by a Decrease of the Platelet Count.

According to their influence on the blood platelet count, the cases in Table 2 may be divided into 2 groups :-

1. Group of infantile diseases with no influence on the platelet count. Hypotrophy, eczema seborrhoicum and dyspepsia levis.

2. Group of infantile diseases accompanied by a decrease of the platelet count. Influenza, rhinopharyngitis, pharyngitis and bronchitis.

Of course, sick babies who were more or less in a serious condition were excluded. Thus, the change of blood platelet of 14 cases in which babies were expected to be in B-avitaminosis were shown in Table 2 (Cf. Table 2).

Blood was examined 4 times in one case (No. 10 in Table 2), 3 times in 6 cases (Nos. 4, 6, 8, 9, 13 and 14 in Table 2) with an interval of 7-10 days on principle.

In 11 cases among all 14 cases Arakawa's reaction was completely or almost completely negative on the first examination.

In the group of diseases with no influence on the platelet count, platelet count showed a decrease without exception reaching normal limits in 5 cases (Nos. 1, 2, 4, 5, and 14 in Table 2) in about 10 days. In one case of them (No. 4 in Table 2) in which blood was examined 3 times repeatedly, platelets returned to normal limits after showing a slight increase and in the other (No. 14 in Table 2) blood platelets decreased gradually in parallelism with an increase in intensity of Arakawa's reaction of their mothers.

And in another group of diseases accompanied by a decrease of platelet count (Nos. 6-13 in Table 2), it will be seen that these babies were possibly in a condition of B-avitaminosis, because in 7 cases out of 8 cases a remarkable increase of platelets was seen even in the period in which thrombopenia ought to have occurred. And in one case (No. 10 in Table 2) blood platelets decreased showing fluctuation of the platelet count.

* or almost no infiuence. 
TABLE 2. Influenee of vitamin $B$ on number and size of blood platelet, influence on the platelet count or in such diseases

\begin{tabular}{|c|c|c|c|c|c|c|}
\hline \multirow{3}{*}{$\begin{array}{l}\text { Case } \\
\text { No. }\end{array}$} & \multirow{3}{*}{ Date } & \multirow{3}{*}{ Name } & \multirow{3}{*}{ Sex } & \multirow{3}{*}{$\begin{array}{c}\text { Age } \\
(M= \\
\text { months, } \\
d=\text { days })\end{array}$} & \multirow{3}{*}{ Diagnosis } & Arakawa's \\
\hline & & & & & & Right \\
\hline & & & & & & $0^{\prime} 1^{\prime} 2^{\prime} 3^{\prime} 4^{\prime} 5^{\prime}$ \\
\hline 1 & $\begin{array}{r}\text { Dec. } 16 \\
27\end{array}$ & K.Y. & 우 & $\begin{array}{ll}71 & d \\
82 & d \\
\end{array}$ & Atrophy & $\begin{array}{l}-----1 \\
---\cdots-1\end{array}$ \\
\hline $\mathbf{2}$ & $\begin{array}{lr}\text { Sep. } 20 \\
\text { Oct. } \quad 2 \\
\end{array}$ & K.I. & $\hat{\delta}$ & $\begin{array}{l}3 \mathrm{~m} \\
4 \mathrm{~m}\end{array}$ & Hypotrophy & $\begin{array}{l}---1 \\
----1\end{array}$ \\
\hline 3 & $\begin{array}{r}\text { Nov. } 8 \\
25\end{array}$ & S. A. & 䓃 & $3 \mathrm{~m}$ & Hypotrophy & $\begin{array}{l}---\cdots- \\
-----1\end{array}$ \\
\hline 4 & $\begin{array}{r}\text { Nov. } 12 \\
18 \\
25\end{array}$ & Y.H. & $\hat{\delta}$ & $9 \mathrm{~m}$ & Hypotrophy & $\begin{array}{l}------ \\
-----\overline{1} \\
---- \pm \pm\end{array}$ \\
\hline 5 & 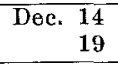 & T.K. & $\hat{\delta}$ & $6 \mathrm{~m}$ & Eczema seborhoicum & $\begin{array}{l} \pm \pm \pm+++ \\
- \pm \pm+++\end{array}$ \\
\hline 6 & $\begin{aligned} & \operatorname{Jan} . 15 \\
& 23 \\
& 28\end{aligned}$ & K.Y. & 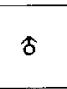 & $7 \mathrm{~m}$ & Rhinopharyngitis & $\begin{array}{l}------ \\
-----\overline{1} \\
---- \pm \pm\end{array}$ \\
\hline 7 & $\begin{array}{l}\text { Dec. } 29 \\
\text { Jan. } 20\end{array}$ & K.I. & 政 & $\begin{array}{l}4 \mathrm{~m} \\
5 \mathrm{~m}\end{array}$ & Rinopharyngitis & $\begin{array}{l}- \pm \pm \pm \pm+ \\
- \pm- \pm \pm+\end{array}$ \\
\hline 8 & $\begin{array}{lr}\text { Jan. } & 6 \\
& 11 \\
\text { Feb. } & 7 \\
\end{array}$ & $\mathrm{~T}, \mathrm{~A}$ & 우 & $8 \mathrm{~m}$ & Influenza & $\begin{array}{l}-----\overline{-} \\
- \pm \pm \pm \pm \pm \\
- \pm \pm+t\end{array}$ \\
\hline 9 & $\begin{array}{r}\text { Jan. } \\
7 \\
17 \\
\end{array}$ & A.I. & t & $9 \mathrm{~m}$ & Influenza & $\begin{array}{l}----- \\
----- \\
- \pm \pm \pm++\end{array}$ \\
\hline 10 & $\begin{array}{r}\text { Jan. } 6 \\
11 \\
18 \\
24 \\
\end{array}$ & T.A. & $\delta$ & $\begin{array}{l}47 \mathrm{~d} \\
52 \mathrm{~d} \\
59 \mathrm{~d} \\
65 \mathrm{~d}\end{array}$ & Pharyngitis & $\begin{array}{l}- \pm \pm \pm \\
-- \pm \pm \pm \pm \\
--- \pm \pm\end{array}$ \\
\hline 11 & $\begin{aligned} \text { Jan. } 14 \\
23\end{aligned}$ & Y.Y. & ㅇ․ & $\begin{array}{l}25 \mathrm{~d} \\
34 \mathrm{~d} \\
\end{array}$ & Bronchitis & $\begin{array}{l}--\overline{-}-\overline{+} \\
-- \pm \pm+\end{array}$ \\
\hline 12 & $\begin{array}{l}\text { Jan. } 29 \\
\text { Feb. } 10\end{array}$ & K.N. & 古 & $13 \mathrm{~m}$ & Bronchitis & $\pm-\bar{t} \pm \pm \pm+4$ \\
\hline 13 & 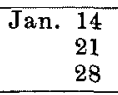 & S. M. & 今 & $5 \mathrm{~m}$ & $\begin{array}{l}\text { Bronchitis with } \\
\text { dyspepsia }\end{array}$ & $\begin{array}{l}-1 \\
------ \\
------ \pm\end{array}$ \\
\hline 14 & $\begin{array}{lr}\text { Sep. } & 28 \\
\text { Oct. } & 5 \\
& 9\end{array}$ & N.W. & $q$ & $\begin{array}{l}13 \mathrm{~m} \\
14 \mathrm{~m}\end{array}$ & $\begin{array}{l}\text { Dyspepsia with } \\
\text { decomposition }\end{array}$ & $\begin{array}{l}- \pm--\overline{-} \\
- \pm+H+H \\
\pm+H+H\end{array}$ \\
\hline
\end{tabular}

* Vitamin $B(+)=$ has been taking vitamin $B$.

Liq. Ory. =Liquor Oryzanin, Ory.=Oryzanin, Liq. Berib.=Liquor Beriberol,

As to the change of size of blood platelets, at the first examination a percentage of large platelets was over $5 \%$ in 7 cases (Nos. 1, 2, $8,11,13$ and 14 in Table 2), and except for No. 2 the percentage of large platelets returned to normal limits at the second examination. In 6 cases out of all the cases (Nos. 3, 5, 6, 8, 12 and 14) the ratio of small: middle: large returned to normal, while in the remaining 7 cases 
red cell count and A rak a wa's reaction in suoh discases as with no as accompanied by a decrease of the platelet count.

\begin{tabular}{|c|c|c|c|c|c|c|}
\hline reaction & I & & Blood p & selets & & Administration \\
\hline Left & & $\begin{array}{c}\text { Total } \\
\text { count per }\end{array}$ & & assificati & & \\
\hline $0^{\prime} 1^{\prime} 2^{\prime} 3^{\prime} 4^{\prime} 5^{\prime}$ & sands) & $\begin{array}{l}\text { (thou- } \\
\text { sands) } \\
\end{array}$ & Small & Middle & Large & $\begin{array}{l}\text { Per os daily } \\
\text { (grs.) }\end{array}$ \\
\hline $\begin{array}{l}--1-1 \\
--1-1\end{array}$ & $\begin{array}{l}4,460 \\
4,160\end{array}$ & $\begin{array}{l}429 \\
296 \\
\end{array}$ & $\begin{array}{l}25 \\
30 \\
\end{array}$ & $\begin{array}{l}69 \\
70 \\
\end{array}$ & $\begin{array}{l}6 \\
0 \\
\end{array}$ & Berib. $\quad 2.0$ \\
\hline $\begin{array}{l}-----\overline{1} \\
----- \pm\end{array}$ & $\begin{array}{l}3,240 \\
3,330 \\
\end{array}$ & $\begin{array}{l}663 \\
263 \\
\end{array}$ & $\begin{array}{l}26 \\
26 \\
\end{array}$ & $\begin{array}{l}64 \\
68 \\
\end{array}$ & $\begin{array}{r}10 \\
6 \\
\end{array}$ & B. $p$ \\
\hline $\begin{array}{l}----\overline{-} \\
--- \pm \pm\end{array}$ & $\begin{array}{l}3,700 \\
3,950 \\
\end{array}$ & $\begin{array}{l}418 \\
390\end{array}$ & $\begin{array}{l}22 \\
24 \\
\end{array}$ & $\begin{array}{l}74 \\
72\end{array}$ & $\begin{array}{l}4 \\
4\end{array}$ & Liq. Para. 5.0 \\
\hline $\begin{array}{l}-----\cdots \\
-----\overline{-} \\
---- \pm \pm\end{array}$ & $\begin{array}{l}5,480 \\
6,100 \\
5,330\end{array}$ & $\begin{array}{l}400 \\
440 \\
250\end{array}$ & $\begin{array}{l}22 \\
23 \\
28\end{array}$ & $\begin{array}{l}71 \\
76 \\
72\end{array}$ & $\begin{array}{l}7 \\
1 \\
0\end{array}$ & Berib. \\
\hline $\begin{array}{l} \pm++4+4 \\
--- \pm \pm \pm\end{array}$ & $\begin{array}{l}4,930 \\
4,940\end{array}$ & $\begin{array}{l}362 \\
316 \\
\end{array}$ & $\begin{array}{l}25 \\
24 \\
\end{array}$ & $\begin{array}{l}74 \\
75 \\
\end{array}$ & 1 & $\begin{array}{ll}\text { Ebios } & 0.5 \\
\text { B. p. } & 0.08 \\
\end{array}$ \\
\hline $\begin{array}{l}- \pm \pm \pm+t \\
\pm \pm- \pm \pm \\
\pm++H+H\end{array}$ & $\begin{array}{l}4,900 \\
5,120 \\
5,640\end{array}$ & $\begin{array}{l}330 \\
280 \\
226\end{array}$ & $\begin{array}{l}28 \\
25 \\
19\end{array}$ & $\begin{array}{l}71 \\
74 \\
80\end{array}$ & $\begin{array}{l}1 \\
1 \\
1\end{array}$ & $\begin{array}{ll}\text { Liq. Ory. } & 5.0 \\
\text { B. p. } & 0.03\end{array}$ \\
\hline $\begin{array}{l}- \pm \pm \pm \pm+ \\
- \pm++H+\end{array}$ & $\begin{array}{l}4,830 \\
4,220\end{array}$ & $\begin{array}{l}385 \\
283\end{array}$ & $\begin{array}{l}30 \\
25\end{array}$ & $\begin{array}{l}67 \\
73\end{array}$ & $\begin{array}{l}3 \\
2\end{array}$ & B. p. \\
\hline $\begin{array}{l}-\overline{-}-\overline{-} \\
- \pm \pm \pm \pm \pm \\
- \pm+++\end{array}$ & $\begin{array}{l}5,370 \\
6,350 \\
5,150\end{array}$ & $\begin{array}{l}507 \\
342 \\
267\end{array}$ & $\begin{array}{l}16 \\
28 \\
24\end{array}$ & $\begin{array}{l}77 \\
68 \\
73\end{array}$ & $\begin{array}{l}7 \\
4 \\
3\end{array}$ & B. $p$. \\
\hline $\begin{array}{l}------ \\
\overline{-}-\overline{-}-\overline{-} \\
++\mathrm{H}+\mathrm{H}\end{array}$ & $\begin{array}{l}5,100 \\
5,450 \\
6,600\end{array}$ & $\begin{array}{l}563 \\
510 \\
412 \\
\end{array}$ & $\begin{array}{l}- \\
- \\
-\end{array}$ & $\begin{array}{l}- \\
- \\
-\end{array}$ & $\begin{array}{l}- \\
-\end{array}$ & Liq. Berib. 5.0 \\
\hline $\begin{array}{l}-- \pm- \pm \pm \\
- \pm-- \pm \pm \\
- \pm- \pm \pm+1\end{array}$ & $\begin{array}{l}4,780 \\
4,690 \\
4,670 \\
4,630\end{array}$ & $\begin{array}{l}300 \\
210 \\
291 \\
219\end{array}$ & $\begin{array}{l}22 \\
25 \\
30 \\
27\end{array}$ & $\begin{array}{l}74 \\
74 \\
70 \\
73\end{array}$ & $\begin{array}{l}4 \\
1 \\
0 \\
0\end{array}$ & 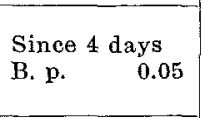 \\
\hline $\begin{array}{l}-----\overline{-} \\
--\cdots \pm \pm\end{array}$ & $\begin{array}{l}5,100 \\
4,920 \\
\end{array}$ & $\begin{array}{l}289 \\
320 \\
\end{array}$ & $\begin{array}{l}24 \\
32 \\
\end{array}$ & $\begin{array}{l}71 \\
66 \\
\end{array}$ & $\begin{array}{l}5 \\
2 \\
\end{array}$ & 0.03 \\
\hline $\begin{array}{l}--\overline{+}-\overline{+} \\
- \pm+1\end{array}$ & $\begin{array}{l}5,250 \\
4,500\end{array}$ & $\begin{array}{l}703 \\
343 \\
\end{array}$ & $\begin{array}{l}19 \\
23 \\
\end{array}$ & $\begin{array}{l}77 \\
74 \\
\end{array}$ & $\begin{array}{l}4 \\
3 \\
\end{array}$ & Liq. Ory. 5.0 \\
\hline $\begin{array}{l}-----\cdots \\
----- \\
---\cdots\end{array}$ & $\begin{array}{l}5,450 \\
4,400 \\
5,550\end{array}$ & $\begin{array}{l}611 \\
370 \\
281\end{array}$ & $\begin{array}{l}14 \\
30 \\
25\end{array}$ & $\begin{array}{l}80 \\
69 \\
74\end{array}$ & $\begin{array}{l}6 \\
1 \\
1\end{array}$ & $\begin{array}{l}\text { B. p. } \quad 0.05 \\
\text { Liq. Abery } 5.0\end{array}$ \\
\hline $\begin{array}{l}--\overline{-} \\
--\overline{-} \\
- \pm+\mathrm{H}+\mathrm{H}\end{array}$ & $\begin{array}{l}5,780 \\
6,100 \\
5,680\end{array}$ & $\begin{array}{l}422 \\
301 \\
251\end{array}$ & $\begin{array}{l}31 \\
35 \\
24\end{array}$ & $\begin{array}{l}64 \\
63 \\
75\end{array}$ & $\begin{array}{l}5 \\
2 \\
1\end{array}$ & $\begin{array}{l}\text { B. p. } \quad 0.03 \\
\text { Liq. Berib. } 10.0\end{array}$ \\
\hline
\end{tabular}

Berib.=Beriberol, B. p. = Beriberolum purum, Liq. Para.=Liquor Paranutrin.

small platelets remained over normal count. It is interesting that Bavitaminosis was of a slight degree in No. 6 , because in this case an increase of small platelets was seen and large platelets had not yet increased at the first examination, and the ratio of platelets returned to normal after 2 weeks. And in No. 3 in which vitamin B had not been administered to the mother, both A raka wa's reaction and blood plate- 
Table 2. Continued.

\begin{tabular}{|c|c|c|c|c|c|}
\hline \multicolumn{3}{|l|}{ of vitamin $\mathrm{B} *$} & \multirow{2}{*}{\multicolumn{2}{|c|}{$\begin{array}{c}\text { Subcutaneous injec- } \\
\text { tion of yakriton } \\
\text { (R.A.U.) } \\
\text { every other day }\end{array}$}} & \multirow{3}{*}{ Remarks } \\
\hline sucklings & \multicolumn{2}{|c|}{ To mothers } & & & \\
\hline $\begin{array}{c}\text { By injection } \\
\text { every other day }\end{array}$ & $\begin{array}{c}\text { Per os daily } \\
\text { (grs.) }\end{array}$ & $\begin{array}{c}\text { By injection } \\
\text { every other day }\end{array}$ & $\begin{array}{c}\text { To } \\
\text { sucklings }\end{array}$ & $\begin{array}{c}\text { To } \\
\text { mothers }\end{array}$ & \\
\hline Ory. $0.05 \mathrm{mg}$. & - & Paranutrin 5.0 & $1 / 2$ & $1 / 2$ & \\
\hline- & Liq. Oryz. 20.0 & - & - & - & : \\
\hline- & - & - & $1 / 2$ & - & \\
\hline Spelzon $\quad 3.0$ & Liq. Ory. 20.0 & $\begin{array}{l}- \\
- \\
-\end{array}$ & $1 / 2$ & $\overline{-}$ & \\
\hline- & Liq. Ory. 30.0 & - & - & - & \\
\hline- & $\begin{array}{l}\text { Liq. Ory. } 20.0 \\
\text { Liq. Berib. } 20.0\end{array}$ & $\begin{array}{r}\text { Orgzanin } \\
\text { mg. }\end{array}$ & $1 / 2$ & - & \\
\hline- & Liq. Ory. 20.0 & - & - & - & \\
\hline $\begin{array}{l}- \\
-\end{array}$ & Vitamin $B(+)$ & $\begin{array}{l}- \\
- \\
-\end{array}$ & $1 / 2$ & $\begin{array}{l}- \\
- \\
-\end{array}$ & $\begin{array}{l}\text { Almost } \\
\text { recovered }\end{array}$ \\
\hline Paranutrin 5.0 & $\begin{array}{l}\text { Liq. Ory. } 20.0 \\
\text { Liq. Berib. } 20.0\end{array}$ & $\begin{array}{l}- \\
- \\
\end{array}$ & $1 / 2$ & & \\
\hline $\begin{array}{l}- \\
- \\
- \\
\end{array}$ & Liq. Ory. 20.0 & $\begin{array}{l}- \\
- \\
-\end{array}$ & $1 / 4$ & $1 / 2$ & Recovered \\
\hline - & $\overline{-}$ & - & $1 / 2$ & $1 / 2$ & \\
\hline - & $\begin{array}{l}\text { Liq. Ory, } 20.0 \\
\text { Liq. Berib. } 20.0\end{array}$ & & $1 / 2$ & $1 / 2$ & \\
\hline $\begin{array}{l}- \\
- \\
-\end{array}$ & Vitamin $B(+)$ & $\underline{-}$ & $1 / 2$ & $\begin{array}{l}- \\
- \\
-\end{array}$ & \\
\hline- & Liq. Ory. 30 & $\begin{array}{l}- \\
- \\
-\end{array}$ & $1 / 2$ & $\overline{-}$ & \\
\hline
\end{tabular}

let did not show a remarkable change in number.

III. Influence of Vitamin B on Blood Platelets in a Healthy Infant and a Healthy Adult.

In both cases of a healthy infant and a healthy adult, not a change of platelets was seen even on an administration of a large amount of vitamin $B$ by way of subcutaneous injection. 
TABLE 3.

Influence of vitamin $B$ an blood platelets in a healthy infant and $a$ healthy adult.

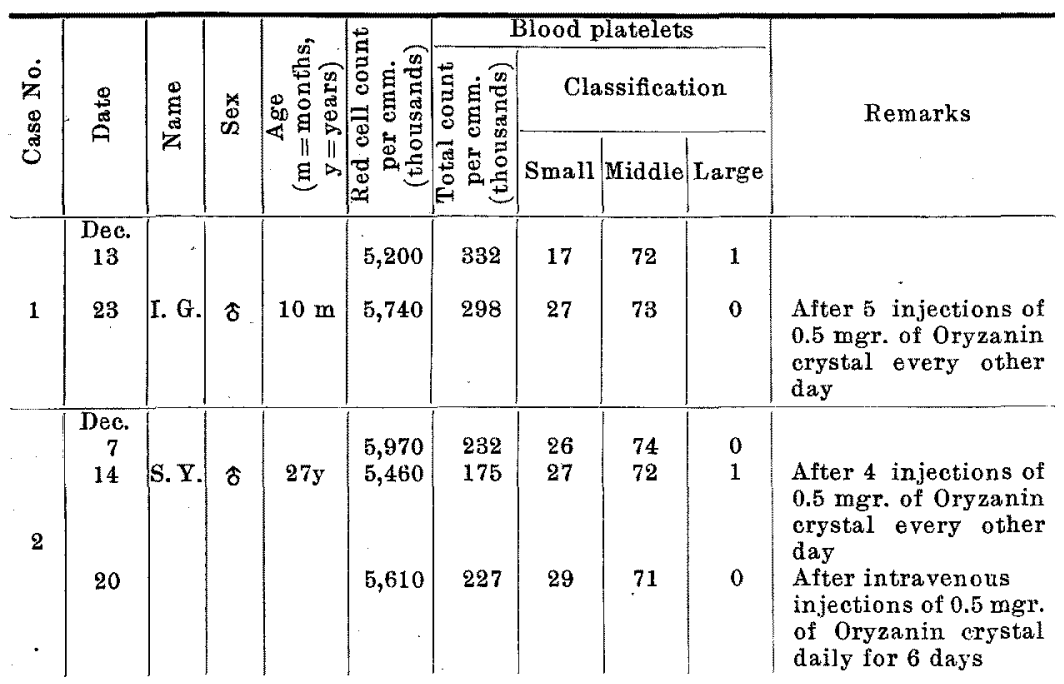

\section{Comment.}

In human B-avitaminosis and beriberi an increase of blood platelets occurs. Kimura ${ }^{11) 12)}$ showed already on the basis of an increase of blood platelet count that most of "healthy" mothers secreting human milk negative to Arakawa's reaction are in a state of avitaminosis B. And in my preceding paper $I^{1)^{2}}$ 3) have shown from an increase of blood platelets that infants fed on human milk negative to Arakawa's reaction are more or less inclined to a state of B-avitaminosis.

Now Nakamura ${ }^{13)}$ reported that blood platelets decreased gradually on vitamin $B$ administration in 14 cases of beriberi in adults. Sarai ${ }^{14)}$ said that in beriberi blood platelets decreased once at the beginning of treatment and showed an increase at one time, then decreasing again till they attained the normal in the convalescence. Accord-

11) J. Kimura, Tohoku J. Exp. Med., 1934, 23, 494.

12) J. Kimura, Tohoku J. Exp. Med., 1935, 27, 360.

13) M. Nak a m u ra, Nippon Naika Gakkai Zasshi, 1925, 13, 317.

14) S. S a rai, Rinsho Byorigaku Ketsuekigaku Zasshi, 1934, 3, 626. 
ing to Iijima, ${ }^{15)}$ an increased platelet count in beriberi returned to normal after decreasing to subnormal count in the convalescent period. In the cases of infantile beriberi $\mathrm{Kohda^{16 } )}$ said that blood picture returned to normal and that especially platelets showed a remarkable decrease when they were treated with vitamin $B$. In the third report of Kimura ${ }^{17}$ he showed that mothers with a weak or negative Arakawa's reaction showed a decrease of platelets on an administration of vitamin $B$.

From the results above mentioned and my own result of 345 cases in which $\mathrm{I}^{2)}$ pointed out that when infants and/or their mothers had been taking vitamin $B$ preparation, platelet count was within normal limits even in the Arakawa-negative group, it will be expected that platelet count of infants fed on human milk weakly or completely negative to Arakawa's reaction will decrease on an administration of vitamin $B$. And the results were as was expected. It can be said, therefore, that high platelet count in infants fed on human milk negative to Arakaw a's reaction was due to avitaminosis $\mathrm{B}$.

As to the decrease of blood platelets in B-avitaminosis, two modes will be seen. In the cases in which an increase of platelets is in high degree platelet count decreased gradually (Cf. Nos. 7, 15 and 18 in Table 1, Nos. 6, 8, 9, 13 and 14 in Table 2), and in the other (Cf. Nos. 2, 5 and 12 in Table 1, Nos. 4 and 10 in Table 2), platelet count became smaller after showing a rise or fall of the count. In these latter cases, the platelet count was not so large, so it is not to be considered that the infants were in a more advanced stage of B-avitaminosis, though Kimura ${ }^{18)}$ said that the increase of blood platelet count became less pronounced in the paralytic stage in the animal experiment.

Port and Akiyama $\mathrm{a}^{19)}$ said that blood platelets decreased more or less during the fever period of acute infectious diseases and they showed a reactive increase with a fall of temperature. According to In ad a and $\mathrm{Kubo^{20 }}$ there were 2 types of influenza. In one of them thrombopenia was seen during fever period and returned to normal gradually, and in the other platelets were within normal limits during fever period and returned to normal after showing a temporary in-

15) Ry. I iji ma, Rinshio Byorigaku Ketsuekigaku Zasshi, 1935, 4, 531.

16) S. Koh d a, Zika Zassi, 1935, No. 420, 607.

17) J. Kimura, Tohoku J. Exp. Med., 1935, 27, 374.

18) J. Kimura, Tohoku J. Exp. Med, 1936, 28, 55.

19) Port and A kiy a ma, Dtsch. Arch. f. Klin. Med., 1912, 106, 362.

20) S. In a d and K. K ubo, Chugai Iji Shimpo, 1921, 453 . 
crease in the convalescent period. Tokuta ${ }^{21)}$ reported that platelets decreased in the fever period of acute infectious disease in general, and showed an increase in the convalescent state. Mc. Lean and Caff $\mathrm{y}^{\mathbf{2 2}}$ said "In ten cases of acute bronchitis, platelet counts were normal with an average of $352,000 . "$

Now at the first blood examination in our own cases of rhinopharyngitis, pharyngitis, influenza and bronchitis etc., a decrease of platelet count was to be seen. However, from the fact that in these diseases platelet count showed a decrease on an administration of vitamin $B$ even in convalescence, it can be said that these infants fed on human milk negative to Arakawa's reaction were in a state of Bavitaminosis. It may be due to an increase of platelets in convalescent period that in 3 cases (Nos. 9, 11 and 12 in Table 2) platelet count remained at the upper limits of the normal count.

As to the classification of platelets, the percentage of large platelets was larger than $5 \%$ in 7 cases out of 16 cases in Table 1 and 6 cases out of 13 cases in Table 2 at the first blood examination. In all the cases, the percentage of large platelets showed a decrease gradually, and then small platelets began to increase and the ratio of platelets returned to normal gradually when they had been treated with vitamin B.

\section{Summary.}

1. In a healthy infant and a healthy adult not a change of blood platelets was seen on an administration of a large amount of vitamin $B$.

2. B-avitaminotic infants fed on human milk negative to A rakawa's reaction showed an anemia and thrombocytosis.

3. When vitamin $B_{i}$ was administered to babies and their mothers, blood platelet count showed a decrease, and anemia began to recover in parallel with an increase in the intensity of A ra kawa's reaction.

4. There were some cases in which platelet count returned to normal in spite of the fact that A rakaw a's reaction of mother's milk remained negative. This is not strange if Taka mats u's ${ }^{10)}$ and others' works are taken into consideration.

5. Blood platelets decrease more rapidly on an administration of vitamin $B$ to both infants and their mothers than on an administration to the former only.

21) K. Tok u ta, Tohoku Igakkai Zasshi, 1925, 8, 512.

22) S. Mc. Lean and J. P. Caffy, Am. J. Dis. Child., 1925, 30, 810. 
6. As to the classification of platelets, large platelets showed a decrease at first and small platelets began to increase and then returned to the normal ratio of platelets on vitamin $B$ administration.

\section{Conclusions.}

In B-avitaminotic infants fed on human milk negative to A ra$\mathrm{kaw}$ a's reaction, an administration of vitamin $B$ makes the increased platelet count smaller and restores the ratio of platelets among themselves to the normal. 